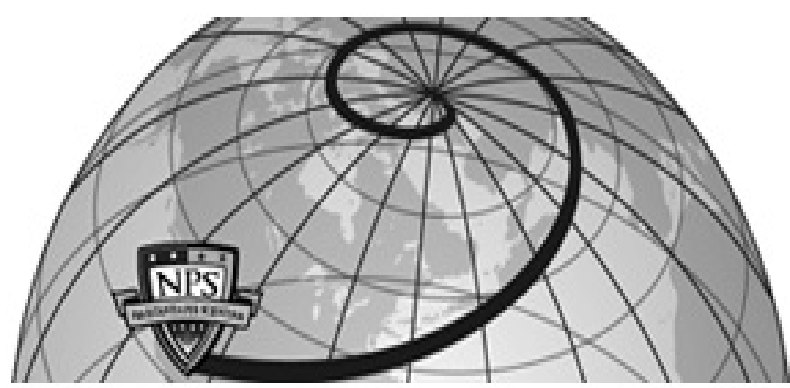

Calhoun: The NPS Institutional Archive DSpace Repository

\title{
Adaptive Filter Techniques for Optical Beam Jitter Control
}

Beerer, M.J.; Yoon, H.; Agrawal, B.N.

SPIE: Acquisition, Pointing, and Laser System Technologies XXIII, Orlando, FL, April 2009. https://hdl.handle.net/10945/34546

This publication is a work of the U.S. Government as definedin Title 17, United States Code, Section 101. As such, it is in thepublic domain, and under the provisions of Title 17, United StatesCode, Section 105, is not copyrighted in the U.S.

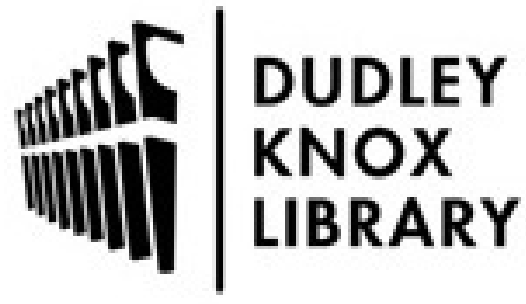

http://www.nps.edu/library
Calhoun is the Naval Postgraduate School's public access digital repository for research materials and institutional publications created by the NPS community. Calhoun is named for Professor of Mathematics Guy K. Calhoun, NPS's first appointed -- and published -- scholarly author.

Dudley Knox Library / Naval Postgraduate School 411 Dyer Road / 1 University Circle Monterey, California USA 93943 


\title{
Adaptive Filter Techniques for Optical Beam Jitter Control
}

\author{
Michael J. Beerer ${ }^{+}$, Hyungjoo Yoon*, and Brij N. Agrawal ${ }^{\S}$ \\ Dept. of Mechanical and Astronautical Engineering \\ Naval Postgraduate School, Monterey, CA 93943
}

\begin{abstract}
The objective of this research is to develop control methods to attenuate laser beam jitter using a fast-steering mirror. Adaptive filter controllers using Filtered-X least mean square and Filtered-X recursive least square algorithms are explored. The disturbances that cause beam jitter include mechanical vibrations on the optical platform (narrowband) and atmospheric turbulence (broadband). Both feedforward filters (with the use of auxiliary reference sensor(s)) and feedback filters (with only output feedback) are investigated. Hybrid adaptive filters, which are a combination of feedback and feedforward, are also examined. For situations when obtaining a coherent feedforward reference signal is not possible, methods for incorporating multiple semi-coherent reference signals into the control law are developed. The controllers are tested on a jitter control testbed to prove their functionality. The testbed is equipped with shakers mounted to the optical platform and a disturbance fast-steering mirror to simulate the effects of atmospheric propagation. Experimental results showed that the feedback adaptive filter controller was superior to the feedforward technique, and the hybrid method achieved the best overall results.
\end{abstract}

Keywords: Optical beam jitter control, adaptive filter, FX-LMS filter, FX-RLS filter

\section{INTRODUCTION}

Optical beam jitter control has become a topic of great interest with applications in directed energy weapons, free-space laser communications and adaptive optics. The objective of this research is to use a fast-steering mirror (FSM) to point a laser beam accurately at a target in the presence of jitter. The disturbances can be time varying and include mechanical vibrations on the optical platform and jitter induced by atmospheric turbulence. Mechanical vibrations caused by rotary or repetitive devices (engines, actuators, electric motors, etc) onboard the platform cause narrowband jitter. Atmospheric induced jitter is spread over a wide range of frequencies causing broadband jitter. A method for attenuating these disturbances must be developed in order to allow high precision optical devices to operate.

Recent work at Naval Postgraduate School (NPS) has focused on adaptive feedforward control. A feedforward adaptive filter may be used only when a reference signal, which is highly correlated with the disturbances, is available. A reference sensor directly measures the disturbance and produces the reference signal. This signal is fed into a transversal filter whose filter gains, or weights, are updated using the error signal (the difference between the beam position and its desired location). In these experiments, the error signal is measured using a beam position sensing detector (PSD). The error signal is used to adaptively update the weights in the filter and generate the control commands for the FSM. Watkins and Agrawal [1]-[2] proposed a feedforward adaptive filter with a filtered-X least mean square (FX-LMS) weight updating algorithm. Yoon et al. [3] proposed a similar feedforward controller using the filtered-X recursive least square (FX-RLS) algorithm and an integrated bias estimator. While the FX-RLS method is computationally more expensive than FX-LMS, Yoon et al. showed that it is superior to FX-LMS in terms of convergence time and steady state performance. Gibson and his research team at UCLA have published several papers, [4]-[8], on beam jitter control using a feedback multichannel recursive least-squares (RLS) lattice filter algorithm.

A disadvantage of any feedforward algorithm is the required reference signal [9]. For good jitter rejection, the reference signal must be correlated with the entire frequency content of the disturbance which may be caused by various sources. Therefore, this "fully coherent" reference signal is often difficult, if not impossible, to obtain. To combat this dilemma,

\footnotetext{
${ }^{+}$Postgraduate student. US Air Force Civilian, Edwards AFB email : michael.beerer@edwards.af.mil

${ }^{*}$ Research associate. Corresponding author: email : (see http://www.drake.googlepages.com)

${ }^{\S}$ Distinguished professor. Director of NPS Spacecraft Research and Design Center: email : agrawal@nps.edu
}

Acquisition, Tracking, Pointing, and Laser Systems Technologies XXIII, edited by Steven L. Chodos, William E. Thompson, Proc. of SPIE Vol. 7338, 733802 - (C) 2009 SPIE · CCC code: 0277-786X/09/\$18 · doi: 10.1117/12.818634 
we develop methods for using multiple "semi-coherent" reference signals in the feedforward control law. In this more realistic situation, signals are available that are only correlated with some component of the total disturbance. This method fuses together information from multiple reference signals to achieve the effect of using a single fully coherent signal. In these experiments, a PSD (primarily measuring the atmospheric disturbance) and an accelerometer (measuring the vibrational disturbance) provide the two reference signals.

For situations where it is not practical to use a feedforward technique, we develop a feedback adaptive filter with the same traversal filter structure as the previously mentioned feedforward filter. In this technique, instead of directly measuring the upstream disturbance (reference sensor), the noise source is internally estimated using only the error signal (feedback signal) and then fed as a reference signal into a feedforward adaptive filter. Finally, a hybrid adaptive filter is introduced that combines both techniques in parallel.

\section{EXPERIMENTAL SETUP}

The jitter control testbed at the Spacecraft Research and Design Center (SRDC), at Naval Postgraduate School (NPS), Monterey, CA was used for this experiment. The testbed contains a laser source, 3-axis accelerometer, beam splitter, two inertial actuators (shakers), two position sensing detectors (PSD, referred to as OT-1 and OT-2) and two fast steering mirrors: a control fast steering mirror (CFSM) and a disturbance fast steering mirror (DFSM). These components are mounted on a floating platform used to simulate a spacecraft/aircraft's vibrational environment. Two shakers are mounted orthogonally to one another to create narrowband vibrations along different axes of the platform. A 3-axis accelerometer is mounted near the shakers to provide signals correlated with the shaker disturbances.

The laser propagates from the source to the DFSM where it is given a broadband disturbance to simulate the effects of atmospheric turbulence. The beam passes onto the vibration platform and to the CFSM where control inputs are applied to the beam. The beam then propagates to the target PSD (OT-2) which is providing an error signal. In order to simulate various beam control scenarios, the target sensor (OT-2) was mounted both on-board and off-board the vibration platform during the experiments. The beam splitter redirects the beam onto the reference signal PSD (OT-1). All of the optics on-board the vibration platform are subjected to the shaker disturbances.

The OT-1 PSD and accelerometer mounted on-board the vibration platform are the reference sensors that provide the multiple reference signals to the feedforward control law. Feedforward adaptive filters have been demonstrated in the past using OT-1 mounted off-board the vibration platform as the sole reference sensor [1]-[3]. In this configuration OT-1 provides a signal reasonably correlated (fully coherent) with both disturbance sources. This scenario, however, may not reflect real spacecraft/aircraft applications. It is more realistic for OT-1 to be mounted on-board the vibration platform. In this position, the sensor continues to provide a signal correlated with the DFSM disturbance, but its correlation with the shaker disturbance is severely degraded. This statement is quantified later in this paper.

The control law is designed in MATLAB Simulink with Real-Time Workshop and XPC Target toolbox. A sample rate of $2 \mathrm{kHz}$ is used throughout the experiment. Cross coupling between the two axes of the CFSM has been shown previously to be negligible [2]. Therefore, we assume they have zero coupling and the control laws are applied independently between the two axes of the mirror.

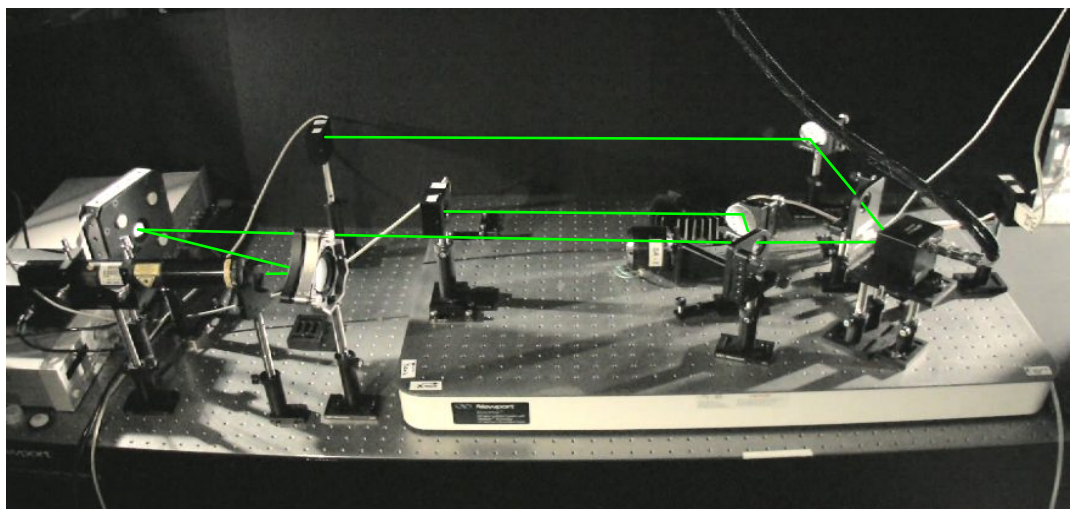

Fig. 1. Experimental setup. OT-1 shown in on-board position, OT-2 shown in off-board position. 


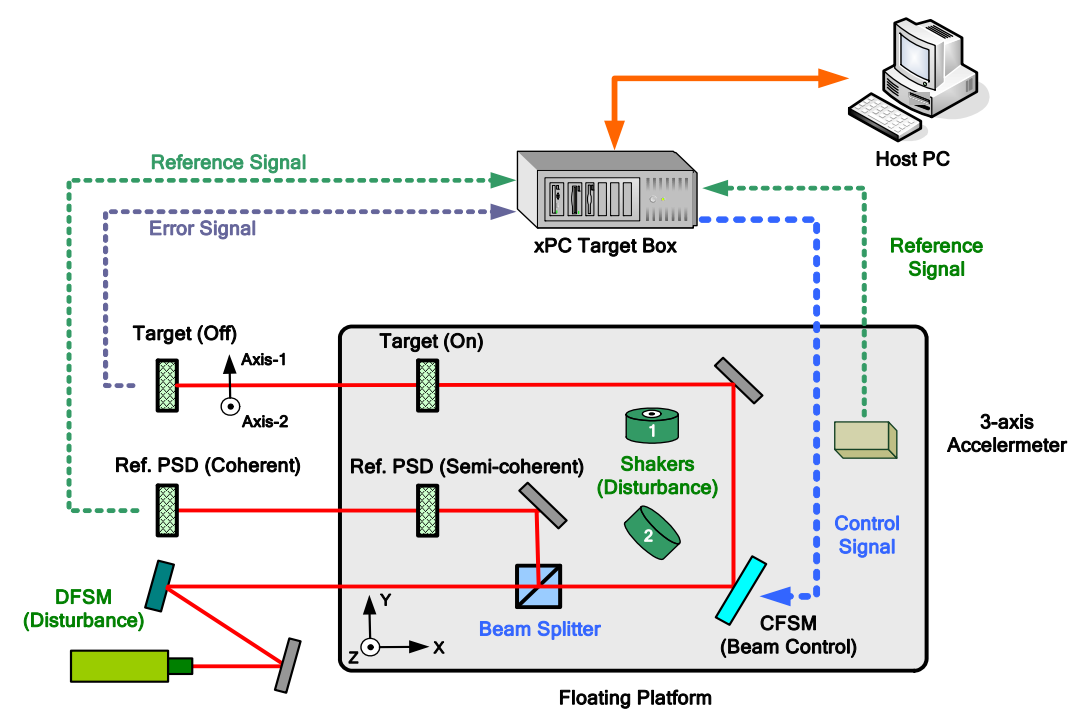

Fig. 2. Experimental setup. OT-1 and OT-2 are shown in both their on-board and off-board positions.

\section{FEEDFORWARD ADAPTIVE FILTERS}

In an adaptive filter, a reference signal (correlated with the disturbance) is input to a transversal filter, consisting of $\mathrm{M}$ stages or weights. The error between the desired beam location at the target and the actual location, e(n), is fed back to the filter to adjust these weights. The output of the transversal filter is the control signal to the CFSM, y(n) [9].

The reference signal, $\mathrm{r}(\mathrm{n})$, is delayed one time step for each of the M stages, forming a vector of delayed inputs, $\mathbf{r}(n)=[r(n), r(n-1), \cdots, r(n-M+1)]^{T} \in R^{M}$. The inner product of the vector of weights $\mathbf{w}(n)=\left[w_{1}(n), w_{2}(n) \cdots, w_{M}(n)\right]^{T} \in R^{M}$ and the reference vector, $\mathbf{r}(n)$, produces the scalar output $y(n)$ :

$$
y(n)=\mathbf{w}^{T}(n) \mathbf{r}(n)
$$

Therefore, the error signal at the position sensor is:

$$
e(n)=d(n)-s(n) * y(n)
$$

Where $d(n)$ is the disturbance and $s(n)$ is the secondary plant dynamics between the CFSM and target sensor (OT-2). The asterisk represents a discrete-time convolution. The goal of the control system is to adaptively update the weighting vector to minimize the error signal, usually represented as the mean square error, $\xi=\mathrm{E}\left[e^{2}(n)\right]$. The LMS and RLS algorithms are methods for updating the weighting vector.

\subsection{Wiener Filter}

The Wiener filter is the optimum linear discrete time filter for estimating the disturbance and requires that the disturbance is both stationary and the spectral properties are known. The Wiener filter is not practical for jitter control because of the unknown and time-varying nature of the disturbance. However, we use the Wiener filter solution as a reference for the best case jitter rejection by the adaptive filter methods under study. The LMS and RLS weight updating algorithms in the following sections approach the optimal Wiener filter weightings. In [10], Haykin shows that the minimum Wiener controlled jitter is:

$$
\xi_{\min }=\sigma_{\min }^{2}=E\left[d^{2}(n)\right]-\mathbf{p}^{\mathrm{T}} \mathbf{R}^{-1} \mathbf{p}
$$

Where $\mathbf{R}$ is the expected value of the autocorrelation matrix of the reference vector, $\mathbf{R}=E\left[\mathbf{r}(n) \mathbf{r}^{\mathrm{T}}(n)\right]$ and $\mathbf{p}$ is the expected value of the cross-correlation vector between the reference vector and the disturbance signal, $\mathbf{p}=E[\mathbf{r}(n) d(n)]$. When the error signal has a zero mean, the mean square error, $\xi$, is equivalent to the variance, $\sigma^{2}$. As a tool for comparison later, we define the ratio between the optimal Weiner disturbance rejection and total disturbance: 


$$
\gamma_{\text {optimal }}=\frac{\sigma_{d}-\sigma_{\text {min }}}{\sigma_{d}}
$$

Where $\sigma_{d}$ is the standard deviation of the beam position at the target with jitter (no control applied). Therefore, $\gamma_{o p t i m a l}$ varies between 0 and $1, \gamma_{\text {optimal }}=0$ for no jitter attenuation and $\gamma_{\text {optimal }}=1$ for absolute attenuation. In order to compare $\gamma_{\text {optimal }}$ to the experimental results, we equivalently define the ratio between the controlled disturbance rejection and the total disturbance:

$$
\gamma_{\text {controlled }}=\frac{\sigma_{d}-\sigma_{\text {controlled }}}{\sigma_{d}}
$$

Where $\sigma_{\text {controlled }}$ is the standard deviation of the beam position at the target with control applied.

\subsection{Filtered-X Least Mean Square Algorithm}

The least-mean-square algorithm (LMS) is on of the simplest adaptive algorithms and has become a standard for comparison with more complex algorithms. The algorithm uses the method of steepest decent to take finite steps toward the optimum weight vector. In [9], Kuo shows that the LMS algorithm to update the weightings is as follows, where $\mu$ is the convergence factor or step size.

$$
\mathbf{w}(n+1)=\mathbf{w}(n)-\mu \mathbf{r}(n) e(n)
$$

The output from the controller, $y(n)$, must pass through the secondary plant between the CFSM and the target sensor (shown in Figure 3). This causes shifts in gain and phase between the error signal and the reference signal. To account for this, we place a copy of the secondary plant transfer function, $\hat{s}(n)$, in the reference signal path to the weight updating algorithm in Equation 6.

$$
\hat{\mathbf{r}}(n)=\hat{s}(n) * \mathbf{r}(n)
$$

This method is referred to as the Filtered-X method in the literature [9]. A system identification was conducted to obtain the secondary plant transfer function using MATLAB's System Identification toolbox and input and output data from the CFSM. Ref. [10] contains a detailed explanation of the system identification methods used.

Finally, we must slightly modify our definition of the reference signal and weighting vector to take into account the presence of a DC component in the error signal. This is referred to as bias estimation [10] and requires the addition of a constant element to the reference signal vector and a corresponding weight to track the bias.

$$
\begin{aligned}
\mathbf{r}_{b}^{T}(n) & =\left[1, \mathbf{r}^{T}(n)\right]=[1, r(n), r(n-1), \cdots, r(n-M+1)] \\
\mathbf{w}_{b}^{T}(n) & =\left[w_{b}(n), \mathbf{w}^{T}(n)\right]=\left[w_{b}(n), w_{1}(n), w_{2}(n), \cdots, w_{M-1}(n)\right]
\end{aligned}
$$

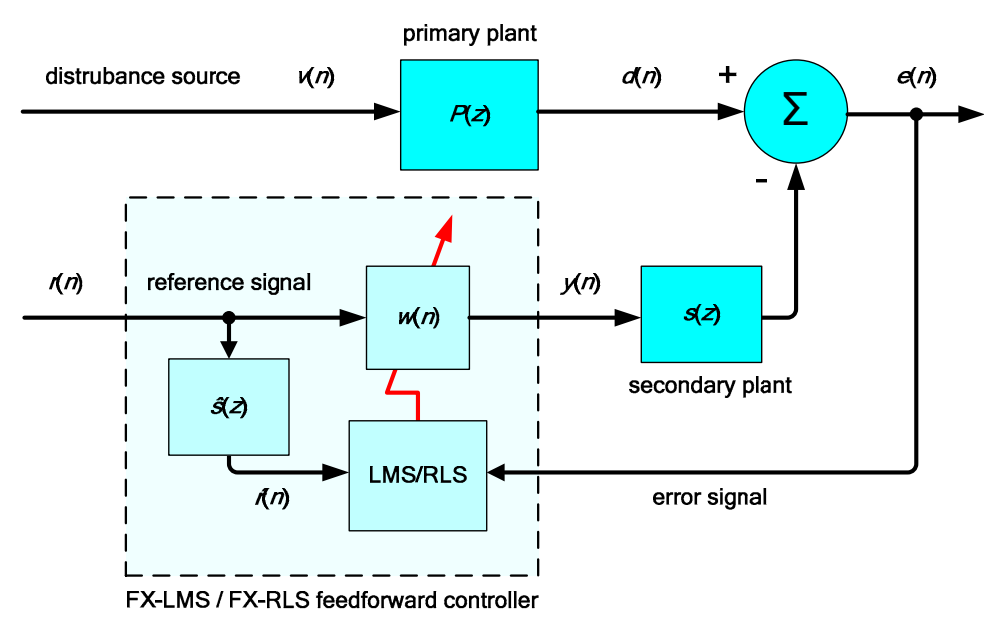

Fig. 3. FX-LMS/FX-RLS implementation. Dark shaded blocks represent actual system dynamics. Control algorithm is shown inside the dashed box. The secondary plant dynamics S(z) and s(n) (from Equation 7) are Z-transform pairs. 


\subsection{Filtered-X Recursive Least Square Algorithm}

The recursive-least-square (RLS) algorithm generally provides faster convergence and smaller steady state error than the LMS algorithm [9]. The main difference is the RLS algorithm's cost function has a memory of errors with a forgetting factor of $0<\lambda \leq 1$, while the LMS cost function does not have memory [9]. The transversal filter structure of the FXRLS algorithm is identical to that of FX-LMS, the difference is the weight updating algorithm. The RLS algorithm to update the weighting vector $\mathbf{w}(n)$ at each instance is the following [9].

$$
\begin{gathered}
\mathbf{k}(n)=\frac{\lambda^{-1} \mathbf{Q}(n-1) \hat{\mathbf{r}}(n)}{1+\lambda^{-1} \hat{\mathbf{r}}^{T}(n) \mathbf{Q}(n-1) \hat{\mathbf{r}}(n)} \\
\mathbf{w}(n)=\mathbf{w}(n-1)+\mathbf{k}^{T}(n) e(n) \\
\mathbf{Q}(n)=\lambda^{-1} \mathbf{Q}(n-1)-\lambda^{-1} \mathbf{k}(n) \hat{\mathbf{r}}^{T}(n) \mathbf{Q}(n-1)
\end{gathered}
$$

Where $\mathbf{k}(n) \in R^{M}$ is the time-varying gain vector, and $\mathbf{Q}(n) \in R^{M \times M}$ is the inverse correlation matrix. See [10] for a more rigorous explanation of the RLS algorithm. Note that the use of $\hat{\mathbf{r}}$ indicates that the reference signal is filtered through the secondary plant dynamics just as in the FX-LMS algorithm.

Also, as in the FX-LMS algorithm, the reference signal and weight vectors are augmented with a constant element to track the DC component of the error signal (Equations 8 and 9). Therefore, the time-varying gain vector and inverse correlation matrix are: $\mathbf{k}(n) \in R^{M+1}$, and $\mathbf{Q}(n) \in R^{(M+1) \times(M+1)}$.

\subsection{Feedforward Adaptive Filters with Multiple Reference Signals}

In [1]-[3], the FX-LMS and FX-RLS algorithms described above have a standard transversal filter structure and use a single-channel reference signal. In this paper, we are provided two semi-coherent reference signals that are each correlated with only a component of the total beam jitter. When using two reference signals, we give distinction between the numbers of accelerometer stages $(M)$ and PSD stages (now denoted as $S$ ).

\subsubsection{Method 1: Summation of filter outputs}

Method 1 uses two separate control blocks (an accelerometer block and a PSD block). The individual outputs are summed and sent to the CFSM. Therefore, the RLS algorithm will manipulate two inverse correlation matrices per axis: $\mathbf{Q}_{\text {Accel }}(n) \in R^{(M+1) \times(M+1)}, \mathbf{Q}_{P S D}(n) \in R^{(S+1) \times(S+1)}$. The RLS algorithm requires on the order of $\mathrm{L}^{2}$ operations per time step, where $\mathrm{L}$ is the filter order [9]. As a result, method 1 requires $\mathrm{O}\left\{(\mathrm{M}+1)^{2}+(\mathrm{S}+1)^{2}\right\}$ operations. A difficulty with parallel adaptive filters is that their performance and characteristics have not been proved mathematically, as opposed to an individual adaptive filter. Placing the adaptive filters in parallel may cause unexpected interactions.
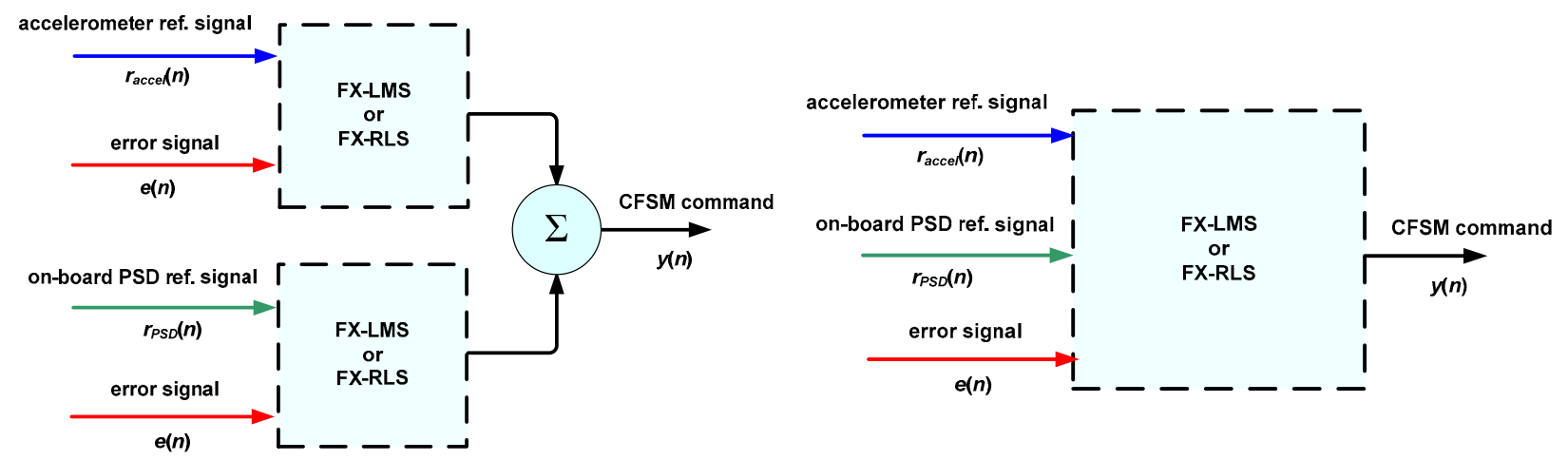

Fig.4. Multiple reference signals using method 1 (left) and method 2 (right). 


\subsubsection{Method 2: Augmentation of reference signals}

For method 2, the reference signals are combined inside a single control block. The reference signal and weight vectors are modified to contain both accelerometer and PSD stages.

$$
\begin{gathered}
\mathbf{r}(n)=\left[1, r_{\text {Accel }}(n), r_{\text {Accel }}(n-1), \cdots, r_{\text {Accel }}(n-M+1), r_{P S D}(n), r_{P S D}(n-1), \cdots, r_{P S D}(n-S+1)\right]^{T} \\
\mathbf{w}(n)=\left[w_{b}(n), w_{1}(n), w_{2}(n), \cdots, w_{M+S}(n)\right]^{T}
\end{gathered}
$$

The rest of the algorithm is implemented as described is section 3.2 and 3.3. Method 2 has a simpler structure compared to method 1 because it only requires one filter. The RLS algorithm will manipulate one very large inverse correlation matrix per axis: $\mathbf{Q}(n) \in R^{(M+S+1) \times(M+S+1)}$ and requires $\mathrm{O}\left\{(\mathrm{M}+\mathrm{S}+1)^{2}\right\}$ operations per time step. Method 2 is, therefore, computationally more expensive than method 1 .

\section{FEEDBACK ADAPTIVE FILTERS}

Feedback adaptive filters use the same single channel transversal filter structure as the feedforward controllers. The error sensor (OT-2 for our experiment) always contains a residual noise signal that is utilized in the feedback adaptive filter to create a reference signal [9]. This techniques is similar to the feedforward controller, however it internally generates its own reference signal using the adaptive filter output, $y(n)$, and the error signal, $e(n)$, as described by Equation 15 [9].

$$
r(n)=\hat{d}(n)=e(n)+\hat{s}(n) * y(n)
$$

Comparing the feedforward and feedback algorithms in Figures 3 and 6, respectively, shows their near identical structure. The reference signal is essentially an estimate of the primary noise source from Equation 2 and, therefore, given the distinction $\hat{d}(n)$ [9]. The secondary plant estimate, $\hat{\mathrm{S}}(z)$, is the same that is employed for the Filtered-X method. Kuo shows that under ideal conditions, when $\hat{\mathrm{S}}(z)=\mathrm{S}(z)$, the feedback method is transformed into the feedforward method [9]. As a result, the performance of the feedback controller compared to the feedforward controller depends on obtaining an accurate secondary plant model with our system identification methods versus the quality of the feedforward reference sensor measurement.

The feedback controller uses the same Filtered-X method and bias estimator as described in the previous sections.

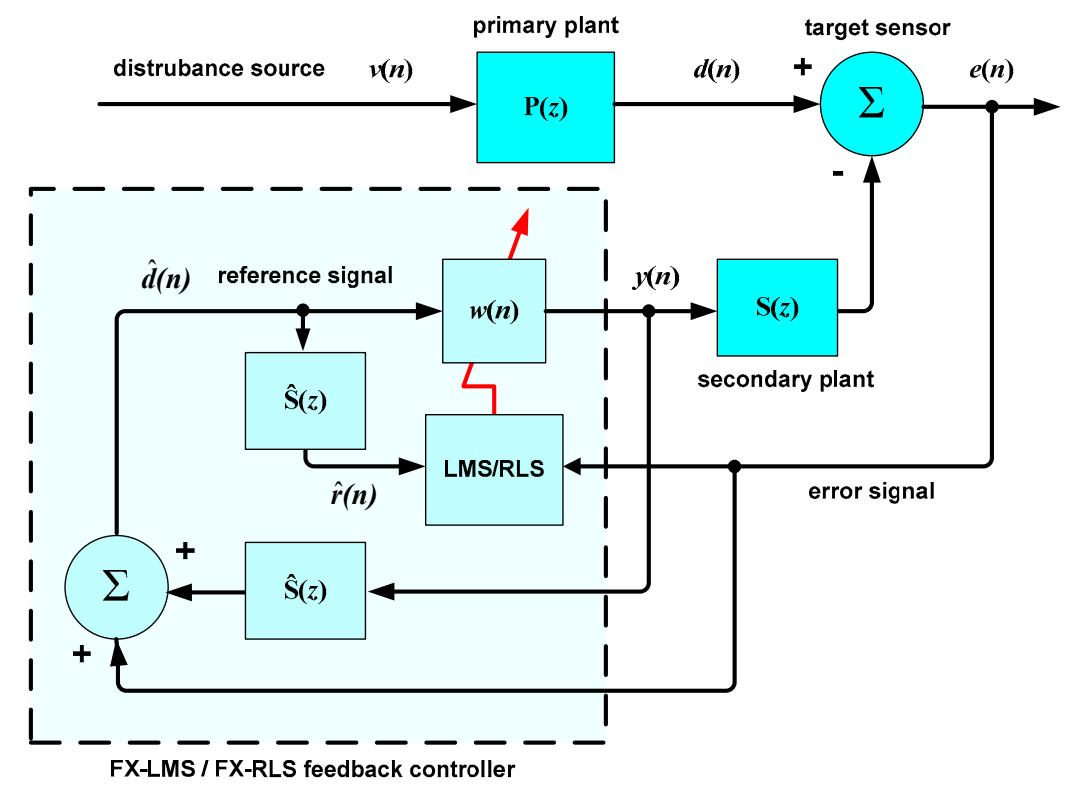

Fig. 5. Feedback adaptive filter 


\subsection{Parallel PI controller}

Initial testing with the feedback controller consisting only of an adaptive filter showed instability when the DC component of the error signal was large. In other words, when the beam was given a large initial bias error in addition to the jitter disturbances, the feedback controller would behave erratically. The internal bias estimator in the adaptive filter seemed to not work as anticipated. However, when the bias error was small, the feedback controller behaved properly.

It is difficult to fully explain this instability; however, it is thought to be due to a large initial transition of the estimated reference signal when the controller is switched on. This theory is based on similar experience with a feedforward adaptive filter with a reference sensor placed downstream of the control actuator. When control was initially applied, and the bias error was corrected, a large DC shift in the reference signal resulted and caused instability. The feedback adaptive filter derives its reference signal from a downstream sensor (error sensor) and, thus, results in a large initial transition in the DC component.

As a solution to improve the robustness of the control method and allow for large DC biases, a proportional-integral (PI) controller was placed in parallel with the adaptive filter. This technique is similar to the feedback adaptive filter research done in [4]-[8], the only difference is our adaptive filters use the more classical transversal filter structure while Gibson et. al employ a lattice structure.

An error PI controller applies fixed gains $\left(K_{p}\right.$ and $\left.K_{i}\right)$ to the error signal and the integral of the error signal. The PI control law is shown in Equation 16 [11].

$$
y_{P I}(n)=K_{p} \cdot e(n)+K_{i} \cdot \int e(n) d n
$$

This classical linear time-invariant control technique works to push the error signal towards zero. With the PI controller placed in parallel with the adaptive filter, it removes the initial bias error so that adaptive filter only must reject the jitter and not the DC bias.

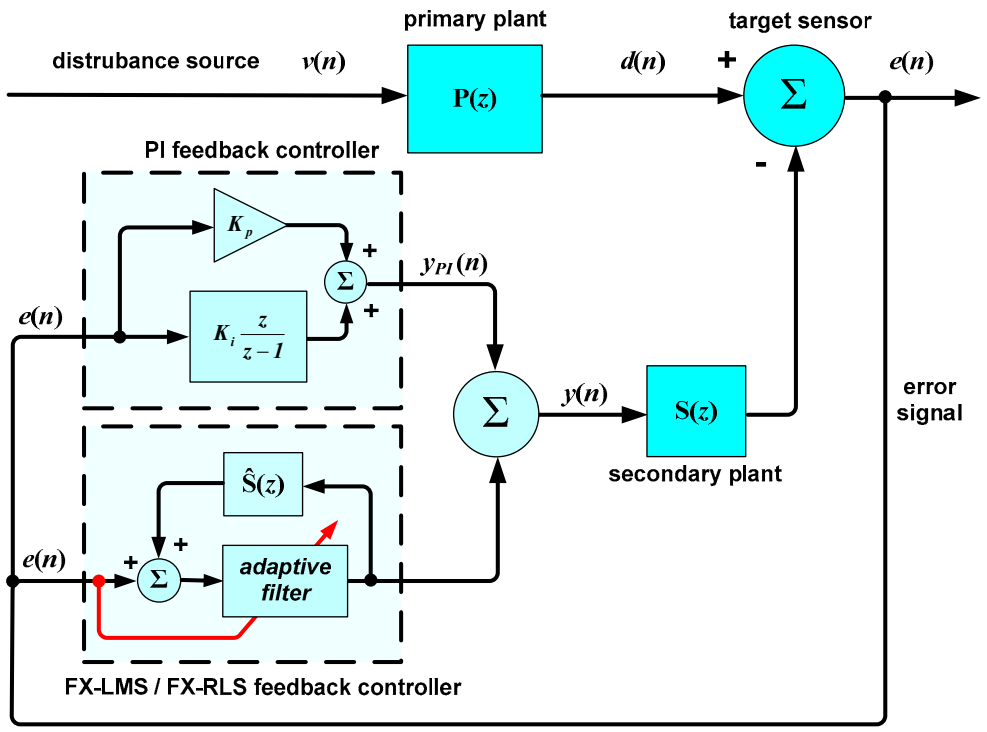

Fig.6. Feedback adaptive filter with parallel PI controller

\subsection{Hybrid adaptive filter}

A combination of the feedback and feedforward methods is refereed to in the literature as a hybrid adaptive filter. To accomplish this we simply place feedback and feedforward adaptive filters in parallel [9]. This method utilizes both the reference signal(s) and the internally generated reference signal. For the same reasons mentioned in the previous section, a PI controller was placed in parallel with the hybrid controller for the experiments. The hybrid controller uses parallel adaptive filters, and therefore, as mentioned earlier, cannot be mathematically analyzed. 


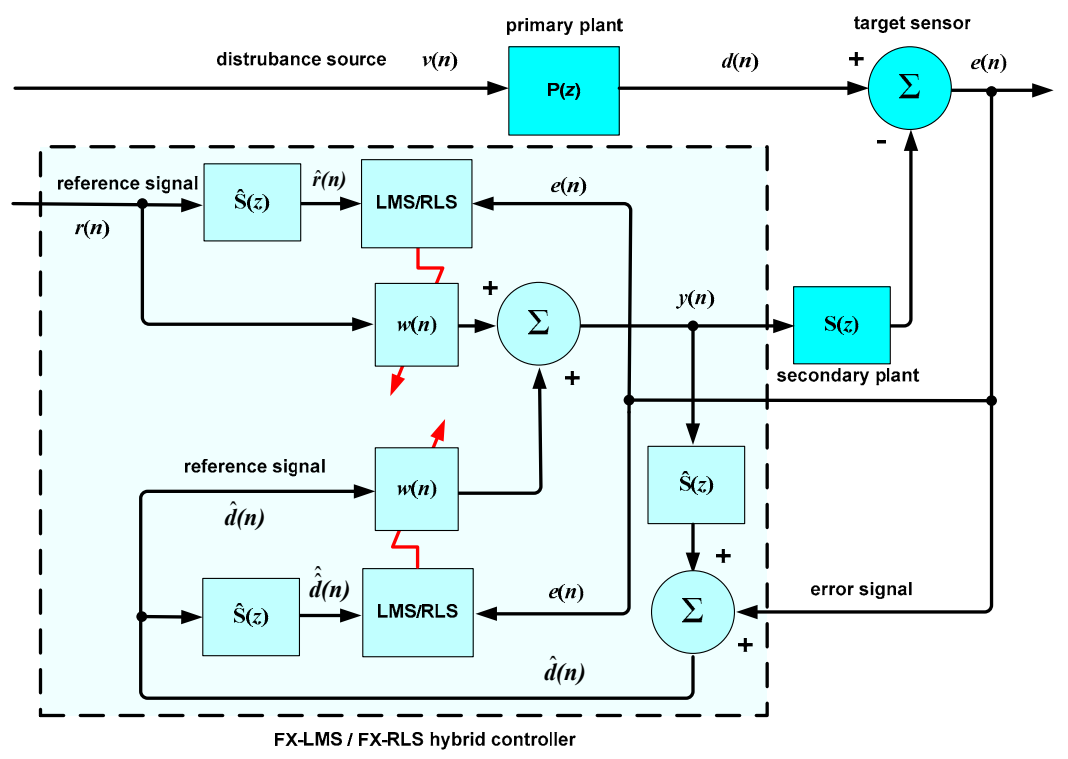

Fig. 7. Hybrid adaptive filter implementation, parallel PI controller not shown.

\section{EXPERIMENTAL RESULTS}

Several experiments with various scenarios were run on the testbed to explore the capabilities of the proposed control techniques. Table 1 summarizes the characteristics of the disturbances and their individual contributions to the beam position error at the target. The path length of beam was approximately 1 meter, therefore $\mu \mathrm{m}$ and $\mu$ radians are essentially interchangeable. The data is reported as the standard deviation, $\sigma$, of the jitter radius. Experiments were conducted with the target sensor in two different positions (on and off the vibration platform). The effects of the disturbances vary between the two positions.

Table 1: Disturbance Characteristics

\begin{tabular}{ccccc}
\hline Target & $\begin{array}{c}\text { Bias } \\
(\mathrm{DC})\end{array}$ & $\begin{array}{c}\text { Narrowband } \\
\text { (Shakers) }\end{array}$ & Broadband (DFSM) & Total Jitter \\
\hline Off-board & $\approx 1000 \mu \mathrm{m}$ & $\begin{array}{l}40 \mathrm{~Hz}, \sigma \approx 40 \mu \mathrm{m} \\
60 \mathrm{~Hz}, \sigma \approx 30 \mu \mathrm{m}\end{array}$ & $0-200 \mathrm{~Hz}, \sigma \approx 51 \mu \mathrm{m}$ band-limited white noise & $\sigma \approx 71 \mu \mathrm{m}$ \\
\hline \multirow{2}{*}{ On-board } & $\approx 1000 \mu \mathrm{m}$ & $\begin{array}{l}40 \mathrm{~Hz}, \sigma \approx 18 \mu \mathrm{m} \\
60 \mathrm{~Hz}, \sigma \approx 17 \mu \mathrm{m}\end{array}$ & $0-200 \mathrm{~Hz}, \sigma \approx 48 \mu \mathrm{m}$ band-limited white noise & $\sigma \approx 52 \mu \mathrm{m}$ \\
\hline
\end{tabular}

\subsection{Reference Signal Correlation Experiment}

In order to give more insight into the performance of the developed control laws an experiment was conducted to characterize the degree of correlation between the various reference signals and the disturbances. Using experimental data from the error and reference sensors, the optimal Wiener jitter rejection, $\gamma_{\text {optimal }}$ from Equation 4 , was calculated comparing each individual reference signal and disturbance source. Therefore, the results in Table 2 represent the best case scenario for a given reference signal. Included in Table 2 is the primary noise estimate from feedback adaptive filter and the fully coherent off-board PSD. 
Table 2: Optimal Wiener jitter rejection, $\gamma_{\text {optimal }}$ from Equation 4

\begin{tabular}{|c|c|c|c|c|c|c|c|c|c|c|c|c|}
\hline $\begin{array}{l}\text { Reference } \\
\text { Signal: }\end{array}$ & \multicolumn{2}{|c|}{$\begin{array}{l}\text { Primary noise } \\
\text { estimate, } \hat{d}(n) \\
45 \text { stgs. }\end{array}$} & \multicolumn{2}{|c|}{$\begin{array}{c}\text { Off-board PSD } \\
\text { (coherent) } \\
45 \text { stgs. }\end{array}$} & \multicolumn{2}{|c|}{$\begin{array}{c}\text { On-board PSD } \\
\text { (semi-coherent) } \\
45 \text { stgs. }\end{array}$} & \multicolumn{2}{|c|}{$\begin{array}{l}\text { Accel. } \\
\text { X-axis } \\
45 \text { stgs. }\end{array}$} & \multicolumn{2}{|c|}{$\begin{array}{l}\text { Accel. } \\
\text { Y-axis } \\
45 \text { stgs. }\end{array}$} & \multicolumn{2}{|c|}{$\begin{array}{l}\text { Accel. } \\
\text { Z-axis } \\
45 \text { stgs. }\end{array}$} \\
\hline \multirow{2}{*}{ PSD Axis: } & \multicolumn{2}{|c|}{ Axis } & \multicolumn{2}{|c|}{ Axis } & \multicolumn{2}{|c|}{ Axis } & \multicolumn{2}{|c|}{ Axis } & \multicolumn{2}{|c|}{ Axis } & \multicolumn{2}{|c|}{ Axis } \\
\hline & 1 & 2 & 1 & 2 & 1 & 2 & 1 & 2 & 1 & 2 & 1 & 2 \\
\hline DFSM & 0.932 & 0.914 & 0.958 & 0.959 & 0.958 & 0.957 & 0.081 & 0.144 & 0.098 & 0.151 & 0.093 & 0.120 \\
\hline Shaker 1 & 0.965 & 0.953 & 0.958 & 0.953 & 0.950 & 0.938 & 0.568 & 0.684 & 0.825 & 0.850 & 0.951 & 0.928 \\
\hline Shaker 2 & 0.953 & 0.947 & 0.954 & 0.946 & 0.624 & 0.933 & 0.923 & 0.893 & 0.915 & 0.893 & 0.953 & 0.913 \\
\hline Both Shakers & 0.951 & 0.954 & 0.951 & 0.944 & 0.636 & 0.889 & 0.553 & 0.663 & 0.875 & 0.853 & 0.951 & 0.933 \\
\hline All Dist. & 0.946 & 0.931 & 0.833 & 0.841 & 0.578 & 0.692 & 0.290 & 0.275 & 0.342 & 0.408 & 0.411 & 0.402 \\
\hline
\end{tabular}

For attenuating all disturbances, the off-board PSD reference signal performs significantly better than the on-board PSD. Also, as expected, the accelerometer reference signals are successful at rejecting the shaker disturbances but completely ineffective for control of the DFSM disturbance.

Due to the two orthogonally mounted shakers, the vibrational disturbance is complex and along all three axes of the platform. As a result, it is difficult to choose a proper signal from the 3-axis accelerometer to use as one of the multiple feedforward reference signals. For this research, the accelerometer signal that produced in best results Table 2 was used. Therefore, the Z-axis accelerometer signal was used for both axes of the control law (Axis-1 and Axis-2). In a more complex system with many sources of vibration this technique may not be appropriate. In such a system, the signals from all three axes of the accelerometer could be incorporated into the control law.

\subsection{Feedforward Adaptive Filter Experiments}

The first series of experiments tested the multiple reference signal feedforward adaptive filters against the single reference signal method from [1]-[3]. Our goal is to show that multiple semi-coherent reference signals can be employed when a obtaining a fully coherent signal is not possible. Both FX-LMS and FX-RLS adaptive filters were tested using both methods 1 and 2 for combining the semi-coherent reference signals. The target sensor (OT-2) was placed both onboard and off-board the platform to imitate various beam control scenarios.

The filter parameters (convergence factor, number of stages, etc.) were determined by trial and error to maximize performance of each control law while staying within the capabilities of the control computer. A RLS forgetting factor of $\boldsymbol{\lambda}=\mathbf{0 . 9 9}$ and LMS convergence factor of $\boldsymbol{\mu}=\mathbf{0 . 5}$ was used throughout all of the experiments. The convergence factor for the LMS method (which uses a finite step method) was chosen to maximize steady jitter rejection at the cost of a longer convergence times. The LMS method also preformed best with a single stage, versus the RLS method which required many stages to achieve maximum performance.

To characterize the performance of the various control laws, $\gamma_{\text {conrolled }}$ from Equation 5, was calculated for each test along with the convergence time of the control law. The convergence time was defined as the time necessary to achieve 99 percent of steady state performance. Results for the feedforward are summarized below in Table 3. 
Table 3: Feedforward Adaptive Filter Experimental Results

\begin{tabular}{cccccccc}
\hline & \multicolumn{3}{c}{ Controller } & \multicolumn{2}{c}{$\begin{array}{c}\text { itter Rejection } \\
\gamma_{\text {conrolled }} \text { (Eq. 5) }\end{array}$} & $\begin{array}{c}\text { Converge } \\
\text { Time }\end{array}$ \\
\cline { 1 - 8 } $\begin{array}{c}\text { Weight } \\
\text { Update }\end{array}$ & $\begin{array}{c}\text { PSD Stgs } \\
\text { (pos) }\end{array}$ & $\begin{array}{c}\text { Accel } \\
\text { Stgs }\end{array}$ & $\begin{array}{c}\text { Trg } \\
\text { Pos }\end{array}$ & Reference Signal & Axis-1 & Axis-2 & \\
\hline FX-LMS & 1 (off) & 0 & Off & Off-board PSD (coherent) & 0.893 & 0.864 & $0.8 \mathrm{~s}$ \\
\hline FX-LMS & 1 (on) & 45 & Off & Multiple (semi-co), method 1 & 0.914 & 0.878 & $12.5 \mathrm{~s}$ \\
\hline FX-LMS & 1 (on) & 45 & Off & Multiple (semi-co), method 2 & 0.892 & 0.842 & $12.5 \mathrm{~s}$ \\
\hline FX-RLS & 55 (off) & 0 & Off & Off-board PSD (coherent) & 0.785 & 0.799 & $0.8 \mathrm{~s}$ \\
\hline FX-RLS & 10 (on) & 45 & Off & Multiple (semi-co), method 1 & 0.895 & 0.919 & $0.6 \mathrm{~s}$ \\
\hline FX-RLS & 10 (on) & 45 & Off & Multiple (semi-co), method 2 & 0.895 & 0.921 & $0.7 \mathrm{~s}$ \\
\hline FX-LMS & 1 (off) & 0 & On & Off-board PSD (coherent) & 0.770 & 0.595 & $0.3 \mathrm{~s}$ \\
\hline FX-LMS & 1 (on) & 45 & On & Multiple (semi-co), method 1 & 0.895 & 0.892 & $6.5 \mathrm{~s}$ \\
\hline FX-LMS & 1 (on) & 45 & On & Multiple (semi-co), method 2 & 0.871 & 0.858 & $8.7 \mathrm{~s}$ \\
\hline FX-RLS & 55 (off) & 0 & On & Off-board PSD (coherent) & 0.771 & 0.606 & $0.3 \mathrm{~s}$ \\
\hline FX-RLS & 10 (on) & 45 & On & Multiple (semi-co), method 1 & 0.885 & 0.900 & $0.7 \mathrm{~s}$ \\
\hline FX-RLS & 10 (on) & 45 & On & Multiple (semi-co), method2 & 0.892 & 0.896 & $0.5 \mathrm{~s}$ \\
\hline
\end{tabular}

The multiple reference signal method preformed as well, or better, than the single fully coherent reference method in terms of steady state jitter rejection. The multiple semi-coherent signals provide more information to the control law than the single reference signal. The performance of method 1 versus method 2 for combining the reference signals was nearly identical. Method 1 is the less computationally expensive technique and is, therefore, considered the superior method. With the FX-LMS weight updating algorithm, multiple reference signals did take significantly longer to converge to a steady state. The results were consistent with the target sensor mounted both on or off the vibration platform.

Along with the tabulated results above, power spectral density (PSD) plots and jitter time history plots were created. A sample of these plots from the FX-RLS off-board experiments is shown in Figure 9.
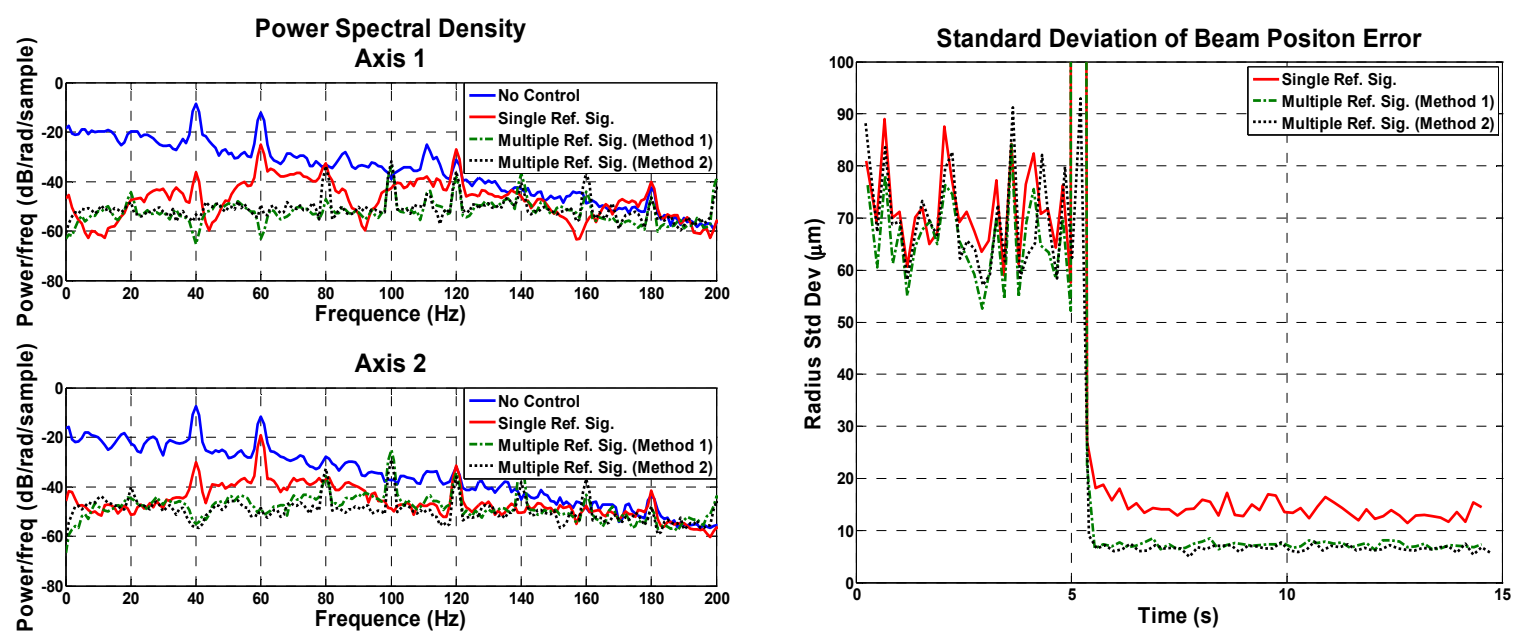

Fig. 8. Comparison of FX-RLS feedforward controllers with off-board target. Control was turned on at $\mathrm{t}=5$ seconds.

\subsection{Feedback and Hybrid Adaptive Filter Experiments}

The second set of experiments tests the feedback and hybrid techniques developed in Chapter 4 to the best performing feedforward method from the previous section. The goal is to verify whether or not using a feedforward reference sensor 
is beneficial. Like in the previous section, the filter parameters were determined by trial and error and the same convergence and forgetting factors were used. The feedback and hybrid methods use a parallel PI controller and the gains were tuned to $\mathbf{K}_{\mathrm{p}}=\mathbf{0 . 0 5}$ and $\mathbf{K}_{\mathrm{i}}=\mathbf{2 0 0}$. The results are tabulated below.

Table 4: Feedback/Hybrid Adaptive Filter Experimental Results

\begin{tabular}{ccccccccc}
\hline \multicolumn{3}{c}{ Controller } & & & \multicolumn{2}{c}{$\begin{array}{c}\text { Jitter Rejection } \\
\gamma_{\text {conrolled }}(\text { Eq. 5) }\end{array}$} & $\begin{array}{c}\text { Converge } \\
\text { Time } \\
\text { (seconds) }\end{array}$ \\
\hline $\begin{array}{l}\text { Weight } \\
\text { Update }\end{array}$ & $\begin{array}{c}\hat{d}(n) \\
\text { Stgs }\end{array}$ & $\begin{array}{c}\text { PSD } \\
\text { Stgs } \\
\text { (pos) }\end{array}$ & $\begin{array}{c}\text { Accel } \\
\text { Stgs }\end{array}$ & $\begin{array}{c}\text { Trg. } \\
\text { Pos }\end{array}$ & Reference Signal & Axis-1 & Axis-2 & \\
\hline FX-LMS & 0 & 1 (on) & 0 & Off & Feedforward & 0.914 & 0.878 & $12.5 \mathrm{~s}$ \\
\hline FX-LMS & 1 & 0 & 0 & Off & Feedback & 0.916 & 0.912 & $3.8 \mathrm{~s}$ \\
\hline FX-LMS & 1 & 1 (on) & 30 & Off & Hybrid & 0.908 & 0.909 & $3.8 \mathrm{~s}$ \\
\hline FX-RLS & 0 & 10 (on) & 45 & Off & Feedforward & 0.895 & 0.921 & $0.7 \mathrm{~s}$ \\
\hline FX-RLS & 50 & 0 & 0 & Off & Feedback & 0.928 & 0.917 & $0.5 \mathrm{~s}$ \\
\hline FX-RLS & 10 & 10 (on) & 30 & Off & Hybrid & 0.935 & 0.931 & $0.4 \mathrm{~s}$ \\
\hline FX-LMS & 0 & 1 (on) & 0 & On & Feedforward & 0.895 & 0.892 & $6.5 \mathrm{~s}$ \\
\hline FX-LMS & 1 & 0 & 0 & On & Feedback & 0.849 & 0.893 & $4.9 \mathrm{~s}$ \\
\hline FX-LMS & 1 & 1 (on) & 30 & On & Hybrid & 0.896 & 0.905 & $6.5 \mathrm{~s}$ \\
\hline FX-RLS & 0 & $10($ on $)$ & 45 & On & Feedforward & 0.885 & 0.900 & $0.7 \mathrm{~s}$ \\
\hline FX-RLS & 50 & 0 & 0 & On & Feedback & 0.897 & 0.887 & $0.3 \mathrm{~s}$ \\
\hline FX-RLS & 10 & $10($ on $)$ & 30 & On & Hybrid & 0.922 & 0.916 & $0.5 \mathrm{~s}$ \\
\hline
\end{tabular}

Testing the various control laws reviled that, overall, the feedback method had superior performance over the feedforward method. The hybrid adaptive filter achieved still better results. This verifies the statement made in Chapter 4 , that depending on the quality of the secondary plant model used to estimate the primary noise source $(\hat{d}(n))$, the feedback method may perform better than feedforward.

\subsection{Conclusion}

Adaptive filter control techniques are very complex and it is difficult to determine performance with classical analytical methods. Verification by experiment is always necessary during the design of these techniques. Such was the case here, where the limiting factor between the performances of feedback versus feedforward adaptive filters was the quality of the secondary plant estimate versus the quality of the disturbance measurement from the reference sensor(s). Both of which are difficult to directly quantify and compare.

The results of the experiments in this paper can be summarized in the following statements: For optical beam jitter control using a FSM, it is viable to employ multiple semi-coherent reference signals in a feedforward adaptive filter when a single fully coherent reference signal is not available. The feedback adaptive filter performs better than feedforward, however, when a feedforward reference sensor(s) is available, it may be beneficial to employ it in a hybrid adaptive filter control scheme.

\section{REFERENCES}

[1] R. J. Watkins and B. N. Agrawal, "Use of least means squares filter in control of optical beam jitter," Journal of Guidance, Control, and Dynamics, vol. 30, no. 4,pp. 1116-1122, July-August 2007.

[2] R. J. Watkins, "The adaptive control of optical beam jitter," Ph.D. dissertation, Naval Postgraduate School, Monterey, CA., December 2004.

[3] H. Yoon, "Laser Beam Jitter Control Using Recursive-Least-Square AdaptiveFilters," Submitted to Optical Engineering. 
[4] Orzechowski, N. Chen, S. Gibson, and T.-C. Tsao, "Optimal jitter rejection in laser beam steering with variableorder adaptive control," in Proceedings of SPIE, vol. 6569, 2007, 65690V.

[5] P. K. Orzechowski, N. Y. Chen, J. S. Gibson, and T.-C. Tsao, "Optimal Suppression of Laser Beam Jitter by HighOrder RLS Adaptive Control," IEEE Transactions on Control Systems Technology, vol. 16, no. 2, pp. 255-267, March 2008.

[6] P. K. Orzechowski, J. S. Gibson, and T.-C. Tsao, "Adaptive Control of Jitter in a Laser Beam Pointing System," in Proceedings of the American Control Conference, Minneapolis, Minnesota, USA, June 2006, pp. 2700- 2705.

[7] N. O. P'erez Arancibia, N. Y. Chen, J. S. Gibson, and T.-C. Tsao, "Variable-order adaptive control of a microelectromechanical steering mirror for suppression of laser beam jitter," Optical Engineering, vol. 45, no. 10, October 2006, 104206.

[8] N. O. P'erez Arancibia, N. Chen, S. Gibson, and T.-C. Tsao, "Adaptive control of jitter in laser beam pointing and tracking," in Proceedings of SPIE, vol. 6304, 2006, 63041G.96S.-B.

[9] S. M. Kuo and D. R. Morgan, Active Noise Control Systems: Algorithms and DSP Implementations. New York: Wiley-Interscience, 1996.

[10] S. Haykin, Adaptive Filter Theory, 4th Ed., Prentice-Hall, Upper Saddle River, NJ,2002.

[11] K. Ogata, Modern Control Engineering, 4th Ed., Prentice-Hall, Upper Saddle River, NJ, 2002. 